\title{
Symmetries and Symmetry Breaking *
}

Willem T.H. van Oers

TRIUMF, 4004 Wesbrook Mall, Vancouver, BC Canada V7H 2Y6

and

Department of Physics and Astronomy, University of Manitoba, Winnipeg, MB, R3T

2N2, Canada

In understanding the world of matter the introduction of symmetry principles following experimentation or using the predictive power of symmetry principles to guide experimentation is most profound. The conservation of energy, linear momentum, angular momentum, charge, and CPT involve fundamental symmetries. All other conservation laws are valid within a restricted subspace of the four interactions: the strong, the electromagnetic, the weak, and the gravitational interaction. In what follows comments will be made regarding parity violation in hadronic systems, charge symmetry breaking in two nucleon and few nucleon systems, and time-reversal-invariance in hadronic systems.

In hadronic parity violation, theory and experiment meet in the weak meson-nucleon couplings as defined, for instance, in the seminal article by Desplanques, Donoghue, and Holstein (DDH). [1] In analogy with the one boson exchange model for the strong N-N interaction, one defines seven weak meson-nucleon couplings for $\pi, \rho$, and $\omega$ exchanges and according the change in isospin. These seven weak meson-nucleon couplings or another set of seven parameters in another theoretical framework characterize the hadronic weak interaction. For a more recent calculation of the weak meson-nucleon couplings see Feldman, Crawford, Dubach, and Holstein. [2] The latest experimental contribution to the determination of the weak meson-nucleon couplings is the measurement at TRIUMF of the longitudinal analyzing power, $A_{z}=\left(\sigma^{+}-\sigma^{-}\right) /\left(\sigma^{+}+\sigma^{-}\right)$, in polarized protonproton scattering at $221 \mathrm{MeV}$, where $\sigma^{+}$and $\sigma^{-}$are the scattering cross sections for positive and negative helicity, respectively. The measurements were performed in transmission geometry with the beam energy and the detector layout selected to ensure that the contribution of the lowest order parity mixing amplitude $\left({ }^{1} S_{0}-{ }^{3} P_{0}\right)$ integrates to zero, hence leaving essentially only a contribution of the $\left({ }^{3} P_{2}-{ }^{1} D_{2}\right)$ parity mixing amplitude. As a consequence the experiment determined the weak meson-nucleon coupling $h_{\rho}^{p p}$. In the experiment the beam current was measured before and after passing a $0.40 \mathrm{~m}$ thick liquid hydrogen target by transverse electric field ionization chambers (TRICs), and the difference determined as function of the helicity state of the incident proton beam. Since many beam parameters may change upon helicity flips and be the cause of false asymmetries, great care was taken to have the sensitivity of the data taking apparatus to the unwanted beam parameters minimized as much as possible, and further to minimize the presence of the unwanted beam parameters, and finally to make corrections to

\footnotetext{
*This work was supported in part by the Natural Sciences and Engineering Research Council of Canada
} 
the data where necessary. Sets of diagnostic, monitoring, and control instrumentation preceded the actual parity data taking apparatus: polarization profile monitors (PPMs) and intensity profile monitors (IPMs), ferrite cored steering magnets, as well as an energy modulating RF cavity. The polarization profile monitors measured on line the unwanted transverse polarization components, $P_{x}$ and $P_{y}$, and their first moments, $y P_{x}$ and $x P_{y}$, across the profile of the beam. The intensity profile monitors measured similarly the intensity across the beam horizontally $(\mathrm{x})$ and vertically $(\mathrm{y})$. The latter allowed to keep the beam position fixed in two places upstream of the parity data taking apparatus. An integral part of the experiment was the optically pumped polarized ion source (OPPIS). Great efforts were made to suppress unwanted helicity correlated changes in the polarized beam extracted from OPPIS. The final result of the $221 \mathrm{MeV}$ experiment is $A_{z}=(0.84 \pm 0.29$ (stat. $) \pm 0.17$ (syst.) $\left.) \times 10^{-7}\right)$. [3] Together with the low-energy proton-proton data at $13.6 \mathrm{MeV}$ [4] and at $45 \mathrm{MeV}$ [5], which determined a linear combination of $h_{\rho}^{p p}$ and $h_{\omega}^{p p}$, for the first time strong constraints could be placed on the acceptable values of both $h_{\rho}^{p p}$ and $h_{\omega}^{p p}$. Many theoretical calculations of $A_{z}$ have been made. Representative calculations are depicted in Fig.1 together with the TRIUMF result (corrected to the $\left({ }^{1} S_{0}-{ }^{3} P_{0}\right)$ zero crossing energy) and the most precise low energy results at 13.6 $\mathrm{MeV}$ and $45 \mathrm{MeV}$. The prediction of Driscoll and Miller [6] is based on the Bonn potential to represent the strong N-N interaction, together with the weak meson-nucleon couplings from Desplanques, Donoghue, and Holstein. The prediction of Iqbal and Niskanen [7] has a $\Delta$ isobar contribution added to the Driscoll and Miller theoretical model on a semi ad-hoc basis. The prediction of Driscoll and Meissner [8] is based on a self-consistent theoretical model, with both weak and strong vertex functions obtained with a chiral soliton approach. Finally, the quark model prediction of Grach and Shmatikov [9] takes explicit account of quark degrees of freedom. None of these predictions are in good agreement with the data, although they have similar shapes due to the energy dependence of the strong interaction phase shifts.

The limits on the weak meson-nucleon couplings $h_{\rho}^{p p}$ and $h_{\omega}^{p p}$ imposed by the low energy results at 13.6 and $45 \mathrm{MeV}$ and by the TRIUMF result at $221 \mathrm{MeV}$ are shown in Fig.2. The error bands $( \pm 1 \sigma)$ are based on a calculation by Carlson et al. [10] assuming the Argonne $v_{18}$ (AV-18) N-N potential, the Bonn 2000 (CD-Bonn) strong interaction coupling constants, and including all partial waves up to $J=8$. Although the TRIUMF measurement does not have a contribution from the $\left({ }^{1} S_{0}-{ }^{3} P_{0}\right)$ parity violating mixing amplitude, and the $\left({ }^{3} P_{2}-{ }^{1} D_{2}\right)$ parity violating mixing amplitude does not contain $h_{\omega}^{p p}$, there is some $h_{\omega}^{p p}$ dependence arising from the higher partial wave mixing amplitudes, i.e., $\left({ }^{1} D_{2}-{ }^{3} F_{2}\right)$. As a result the acceptable band as determined by the TRIUMF experiment is almost perpendicular to those determined by the low energy measurements and greatly reduces the acceptable ranges of both $h_{\rho}^{p p}$ and $h_{\omega}^{p p}$. Adjusting these couplings for the best fit to the three data points, Carlson et al. estimate $h_{\rho}^{p p}=-22.3 \times 10^{-7}$ and $h_{\omega}^{p p}=5.17 \times 10^{-7}$ compared to the DDH "best guess" values of $h_{\rho}^{p p}=-15.5 \times 10^{-7}$ and $h p p_{\omega}=-3.0 \times 10^{-7}$. The solid curve in Fig.1 is calculated using the adjusted couplings.

The current situation regarding the long range weak meson-nucleon coupling $h_{\pi}^{1}$ is not as satisfactory, with large discrepancies between the value extracted from the parity mixed doublet in ${ }^{18} \mathrm{~F}$ in a series of independent experiments and from atomic parity violation 


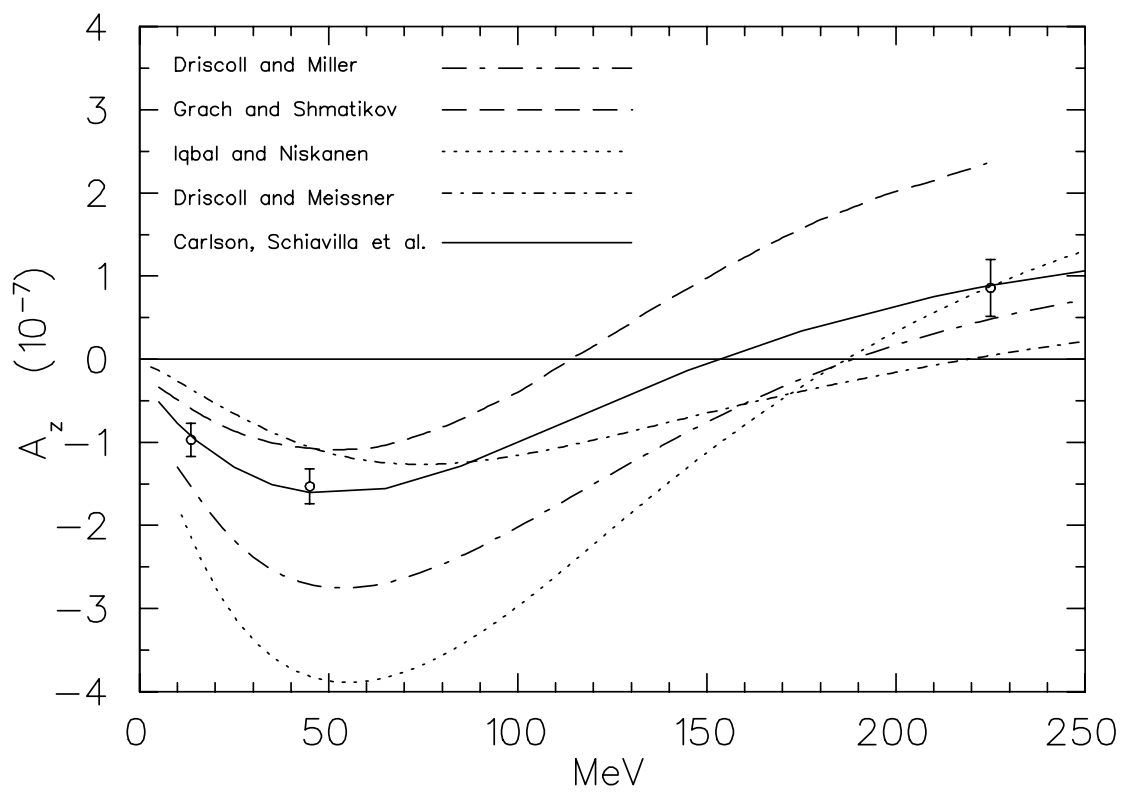

Figure 1. Theoretical predictions for $A_{z}$ and the more precise data at $13.6 \mathrm{MeV}$ (Bonn), $45 \mathrm{MeV}$ (PSI), and $221 \mathrm{MeV}$ (TRIUMF). The solid curve shows the result obtained by Carlson et al. by adjusting the weak meson-nucleon couplings for the best fit to the data.

in $\mathrm{Cs}$ and Tl. A new precision experiment has been mounted at LANL to measure parity violation in polarized cold neutron capture on para-hydrogen. [11] A precise determination of the seven weak meson-nucleon couplings is a prerequisite to verify the many steps of imprinting the weak interaction among mainly strong interacting quarks and gluons. Further experimentation measuring spin rotation of cold neutrons through helium and hydrogen, measuring the asymmetry in photodisintegration of the deuteron with lefthanded and right-handed circularly polarized photons, and measuring the longitudinal analyzing power in polarized proton-proton scattering at $221 \mathrm{MeV}$ with greatly improved precision are essential to precisely determine the seven weak meson-nucleon couplings and to understand the intricacies of hadronic parity violation. A greatly improved 221 $\mathrm{MeV}$ proton-proton parity violation experiment is possible after adding current readout to the counting polarimeters (PPMs), by replacing the transverse electric field ionization chambers (TRICs) by current monitors, and by changing the analog electronics with state-of-the-art digital electronics.

Isospin conservation/charge independence is broken by the mass difference of the 'up' and 'down' quarks and by their electromagnetic interaction. Charge symmetry is a lesser symmetry and corresponds to a rotation in isospin space around the 2-axis. For the neutron-proton system one classifies the interaction as an isospin breaking, chargeasymmetric, and charge-dependent force. Charge symmetry breaking in neutron-proton elastic scattering, manifested through a non-zero value of the difference in the analyzing powers for polarized (unpolarized) neutrons scattered from unpolarized (polarized) protons, is well established. The results of two new charge symmetry breaking experiments will be reported at this Conference. The first one (a TRIUMF experiment) has measured the forward/backward asymmetry in the reaction $n-p \rightarrow d-\pi^{0}$ close to threshold (with a little less than a $2 \sigma$ effect $)\left(A_{f b}=(1.7 \pm 0.55\right.$ (stat. $) \pm 0.8($ syst. $\left.\left.)\right) \times 10^{-3}\right)$. [12] 


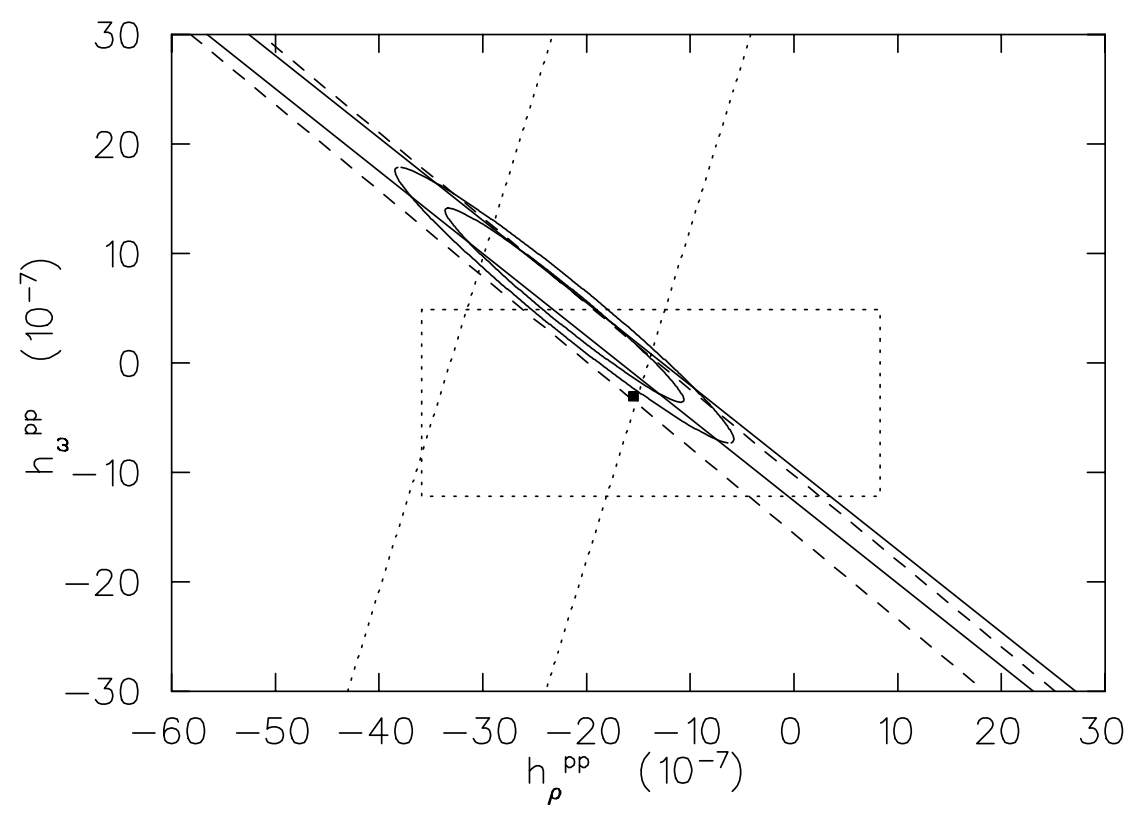

Figure 2. Present constraints on the weak meson-nucleon couplings based on the experimental data and recent calculations by Carlson et al.. The bands are the constraints imposed by the different experiments (13.6 MeV Bonn, dashed; $45 \mathrm{MeV}$ PSI, solid; 221 MeV TRIUMF, dotted. The filled square and dotted rectangle are the DDH "best guess" and "reasonable range", respectively. Also shown are the $68 \%$ and $90 \%$ C.L. contours.

In terms of meson-exchange nucleon-nucleon interaction models, the chief contributions to charge symmetry breaking in this reaction are $\pi-\eta$ mixing and the 'up' and 'down' quark mass difference affecting pion-nucleon scattering. The second one (a IUCF experiment) has measured the reaction $d-d \rightarrow \alpha-\pi^{0}$ just above threshold for $\pi^{0}$ production. Evidence presented for $\pi^{0}$ production in this reaction in an earlier experiment has been controversial. Employing a three-fold coincidence between the $\alpha$-particle and the two $\gamma$ 's from the decay of the $\pi^{0}$, with careful reconstruction of the mass of the latter, determined unambiguously a small cross section for this charge symmetry breaking reaction, in accord with estimates based predominantly on 'up' and 'down' quark mass difference effects $\left(\sigma_{\text {tot }}(228.5 \mathrm{MeV})=12.7 \pm 2.2 \mathrm{pb}\right.$ and $\left.\sigma_{\text {tot }}(231.8 \mathrm{MeV})=15.1 \pm 3.1 \mathrm{pb}\right)$. [13] These charge symmetry breaking experiments test the details of meson-exchange nucleonnucleon interaction models and delineate the contributions from the mass difference of the up and down quarks and their electromagnetic interaction.

Charge symmetry breaking in neutron-proton elastic scattering, mentioned above, also permits an upper limit to be placed on a parity-conserving/ time-reversal-invariancenonconserving nucleon-nucleon interaction mediated by $\rho$ and $a_{1}$-meson exchanges. [14] To greatly decrease the current upper limit on this interaction requires a vastly improved charge symmetry breaking neutron-proton elastic scattering experiment at an energy of $320 \mathrm{MeV}$. At this energy and at the zero-crossing angle of the neutron-proton analyzing power, where $A_{z}$ is measured, the contribution from the uncertain $\rho-\omega$ mixing term is very small. The electromagnetic contribution as well as the neutron proton mass difference affecting $\pi$ and $\rho$ exchanges can be calculated with confidence. Thus from a comparison between experiment and theory one can deduce an improved upper limit on 
a parity-even/time-reversal-odd interaction. From an experimental point of view, however, one prefers a genuine null test of time-reversal-invariance, which is only possible in total cross section measurements (attenuation experiments). Such an experiment is being prepared for execution at COSY. [15] The experiment will measure the parityeven/time-reversal-odd observable $A_{y, x z}$ in transmission geometry. The experiment will use a stored, transversely polarized proton beam (with polarization $P_{y}$ ) and an aligned polarized deuterium target (with polarization $P_{x z}$ ). The tensor polarized deuteron beam is produced in an atomic beam source based on Stern-Gerlach separation in permanent sextupole magnets and adiabetic high frequency transitions. Adequate luminosity is obtained using a windowless storage cell placed on axis of the polarized proton beam from the atomic crossed-beam polarized ion source. For this test of time-reversal-invariance the COSY-ring will function as accelerator, forward angle spectrometer, and detector. Crucial to the experiment is a current monitor, which can measure with the required high precision the decrease in intensity with time as function of the circulating proton beam spin state or the direction of the deuteron tensor polarization. Also of great importance is the precise alignment of the proton beam vector and deuteron beam tensor polarizations in order to suppress unwanted spin correlation coefficients, which could produce a false result. The stated precision of the experiment is one part in $10^{6}$ for $A_{y, x z}$, which would give a sensitivity to $\bar{g}_{\rho}$ of $10^{-3}$ by appropriate choice of the proton beam energy $\left(\bar{g}_{\rho}\right.$ is the time-reversal-invariance nonconserving fraction of the strong $\rho$-meson-nucleon coupling constant). This is a similar upper limit as deduced from the neutron-proton elastic scattering charge symmetry breaking experiments mentioned above. The experiment is currently in an engineering phase. It should be noted that these are the better upper limits on a parity-even/time-reversal-odd interaction in hadronic systems that exist to date. But it should also be noted that there is no room for such an interaction within the Standard Model; it would require flavor non-conservation of the quarks.

\section{REFERENCES}

1. B.Desplanques, J.F.Donoghue, and B.R.Holstein, Ann.Phys.(N.Y.) 124,449(1980).

2. G.B.Feldman, G.A.Crawford, J.Dubach, and B.R.Holstein, Phys.Rev. C43,863(1991).

3. A.R.Berdoz et al., Phys.Rev.Lett. 87,272301(2001).

4. P.D.Eversheim et al., Phys.Lett. B256,11(1991); P.D.Eversheim, private communication (1994).

5. S.Kistryn et al., Phys.Rev.Lett. 58,1616(1987).

6. D.E.Driscoll and G.A.Miller, Phys.Rev. C39,1951(1989); ibid., 40,2159(1989).

7. M.J.Iqbal and J.A.Niskanen, Phys.Rev. C49,355(1994).

8. D.E.Driscoll and Ulf-G.Meissner, Phys.Rev. C41,1303(1990).

9. I.Grach and M.Shmatikov, Phys.Lett. B316,467(1993).

10. J.Carlson, R.Schiavilla, V.R.Brown, and B.F.Gibson, Phys.Rev. C65,035502(2002); R.Schiavilla, private communication(2001).

11. LANSCE experiment NPDGAMMA, J.D.Bowman (spokesperson).

12. E. Korkmaz, these Proceedings.

13. E.J.Stephenson, these Proceedings.

14. M.Simonius, Phys.Rev.Lett. 78,4161(1997).

15. COSY experiment 126, P.D.Eversheim (spokesperson). 